\title{
The Electro-Anatomical Pathway for Normal and Abnormal ECGs in COVID Patients
}

\author{
Peter M van Dam ${ }^{1,3}$, Machteld Boonstra ${ }^{1}$, Rob Roudijk ${ }^{1}$, Marijke PM Linschoten ${ }^{1}$, Emanuela T \\ Locati $^{2}$, Giuseppe Ciconte ${ }^{2}$, Valeria Borrelli ${ }^{2}$, Vincenzo Santinelli ${ }^{2}$, G Vicedomini ${ }^{2}$, MM Monasky $^{2}$, \\ E Micaglio $^{2}$, Luigi Giannelli ${ }^{2}$, Valerio Mecarocci ${ }^{2}$, Zarko Calovic ${ }^{2}$,Carlo Pappone ${ }^{2}$, Peter Loh $^{1}$ \\ ${ }^{1}$ University Medical Center Utrecht, The Netherlands, ${ }^{2}$ IRCCS Policlinico San Donato, Milano, Italy, \\ ${ }^{3}$ ECG Excellence BV, Nieuwerbrug aan den Rijn, Netherlands
}

\begin{abstract}
Patients with COVID-19 frequently have non-typical ECG changes in the QRS and T-wave morphology. The novel CineECG uses using the mean temporal spatial isochrones (mTSI) to relate the activation and recovery pathway to the cardiac anatomy. The aim of this feasibility study is to use the novel CineECG to separate normal from abnormal ECGs.

The ECGs of 100 normal controls were used to obtain the normal mTSI paths values for the QRS, ST segment and $T$-wave. These normal CineECG values were used to classify the COVID-19 ECGs as either as normal or abnormal of 107 patients being treated for COVID-19 in the University Medical Center Utrecht.

The CineECG was able to classify $98 \%$ of the normal ECG correctly and $94 \%$ of the abnormal ECG in comparison to expert ECG classifications.

The ability of the CineECG to relate the ECG to the cardiac anatomy supports the detection of abnormal ECGs. The CineECG might be a novel ECG screening tool to detect potential cardiac involvement of the COVID-19 disease for non-ECG experts.
\end{abstract}

\section{Introduction}

COVID-19 is often associated with cardiovascular complications thereby further increasing the yet huge burden on the health care system [1,2]. The combination of COVID-19 and cardiovascular disease makes that the patient is at high-risk for complications during the treatment. Early recognition of cardiac dysfunction amongst COVID-19 patients is therefore of great importance to optimize for personalized and cardiac treatment. The detection of potential cardiac problems currently requires the involvement of the specialized cardiologist. The ECG of COVID-19 patients with cardiovascular complications show tachycardias, conduction disorders and atypical T-wave morphology changes. The detection of cardiac complications in the COVID-19 population might be improved by the CineECG technology, using the standard 12 lead ECG, relates the ECG waveforms directly to the cardiac anatomy.

This study presents the preliminary results on the detection of deviating ECG waveforms using the CineECG. To determine the normal values for the CineECG, we used 100 normal subjects from the PTB-XL database [3]. The COVID-19 ECGs were obtained from the international Capacity-COVID patient registry. The normal range of specific CineECG parameters were used to classify an ECG from a COVID-19 patient as normal or abnormal.

\section{Aim of the study}

The aim of the study was to reduce the burden on cardiac testing and detect cardiac complications during ICU admission by using CineECG derived from the standard 12 lead ECG.

\section{Methods}

\subsection{Patient Selection}

We selected 121 COVID-19 patients from the CapacityCOVID database (www.capacity-covid.eu). Routine 12lead ECGs (GE Healthcare, MAC5500) were obtained according to standard clinical care during COVID-19 related hospital admissions at the UMC Utrecht. Subjects were retrospectively included in the Capacity-COVID registry and could be excluded via an opt-out procedure. Of all included subjects, raw ECG recordings were exported and used for analysis. The ECGs were reexamined and classified as normal or abnormal by author RR. ECGs classified as both abnormal and not in sinus rhythm were excluded for analysis as well as ECGs where the secondary evaluation did not coincide with the database classification. After exclusion 80 patients were included in the study, with 107 ECGs in total. These 107 ECGs, either normal or abnormal, were further divided in 


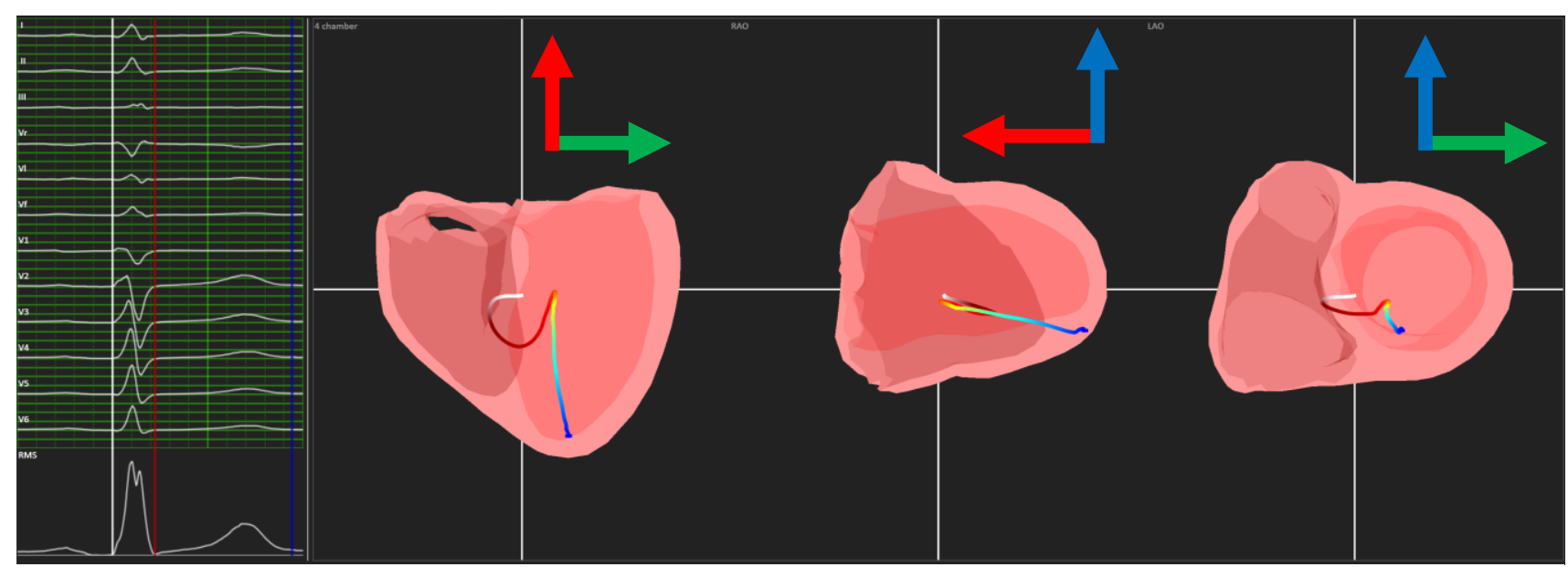

Figure 1:An example of the mTSI of a normal subject from the PTB-XL database. In the panel the 12 lead ECG is shown left with the root mean square signals in the bottom left corner. The QRS onset is indicated by a white line, the QRS end with a red line and the end of the $\mathrm{T}$ wave with a blue line. These colors can also be found in the mTSI shown as a colored line through the ventricles. The ventricles are shown in standard X-ray views: the 4 chamber view (left panel), Right Anterior Oblique (RAO) view (middle panel) and the Left Anterior Oblique view (LAO). The 3 heart axis are shown as red, blue and green arrows. The initial mTSI (white) travels clearly trans-septal (4 chamber view) and moves than to the left chamber (red part), as more ventricular mass is activated on the left. During the ST segment ( yellow/green line) and the T-wave (blue line) the mTSI moves towards the left apex of the heart.

two patient groups based on information from the capacity database: a group with known cardiac history and one without prior to the Covid-19 disease. Of the included patients, 23 patients were known with a cardiac history of conduction disorders or an arrhythmia, 9 patients with heart failure, 25 with coronary artery disease, 7 with a valvular disease, and 7 patients remained unclassified. For 10 patients more than one classification was used.

The data for the normal values was a selection of 100 ECGs labeled as normal from the PTB-XL database [1]. The average age for normal group was $48 \pm 17$ years with $52 \%$ females. For the 80 Covid- 19 patients the average age was $66 \pm 13$ years with $34 \%$ females.

\subsection{The CineECG}

The CineECG computes the mean temporal-spatial isochrones (mTSI), representing the mean activation and recovery pathway of the heart. In this study we will focus on the ventricular activation and recovery, where mTSI was related directly to the ventricular anatomy. Therefore, the CineECG not only needs the 12 lead ECG, but also a model of the heart and the locations of the electrodes on the chest, building the sensor (electrodes) source (ventricles) relationship. The mTSI is computed from the 12 lead ECG in 2 steps [4]. In the first step a vector cardiogram (VCG) is computed using the known torso and limb electrode positions relative to the heart. The VCG thus gives the direction of activation or recovery at any time instant of the ECG. In step 2 of the CineECG algorithm this direction is used to move the mTSI with a predefined velocity. The activation path direction is based on the VCG direction per time sample, using a propagation velocity of $0.7 \mathrm{~m} / \mathrm{s}$ through the myocardium for the QRS and 0.25 for the -Wave [5].

The first point of the mTSI should be chosen close to the initial activation of the heart, which is for normal sinus rhythm on the left septal wall. For this reason, the QRS initiation site of the mTSI is chosen to the septal location closest to the center of ventricular mass.

At last, the computed mTSI was directly related and visualized into the $3 \mathrm{D}$ heart space, using three standard $2 \mathrm{D}$ $\mathrm{X}$-ray views (Figure 1). For the current study the heart and torso model of a 58-year-old male was used [5].

\subsection{ECG and mTSI definitions}

The definition of a normal and abnormal ECG is based on a number of ECG and mTSI definition. In a previous study we used these mTSI parameters to classify an ECG beat as incomplete and complete right bundle branch block ((I)RBBB )[5]. Additional parameters used in this study:

QRST definitions: For each beat the QRS duration is determined, as well as the ST ratio. The ST ratio is defined as the averaged amplitude of ST segment (up to $60 \mathrm{~ms}$ after the QRS end) of the root mean square signal divided by the peak T wave amplitude of the same signal (see Figure 1).

Trans-cardiac ratio (TCR): The trans-cardiac ratio is defined as the 3D-distance between the starting and the ending points of the mTSI during activation, coincident with the QRS onset to the QRS offset. As this measure is influenced by the size of the heart, it was weighted by the size of the heart model, resulting as a relative number.

mTSI direction: The direction of the mTSI, representing the direction of electrical activity, is related to the heart axis. In this study the terminal $S T$ direction $\left(Q R S_{\text {end }}-T\right.$ - 

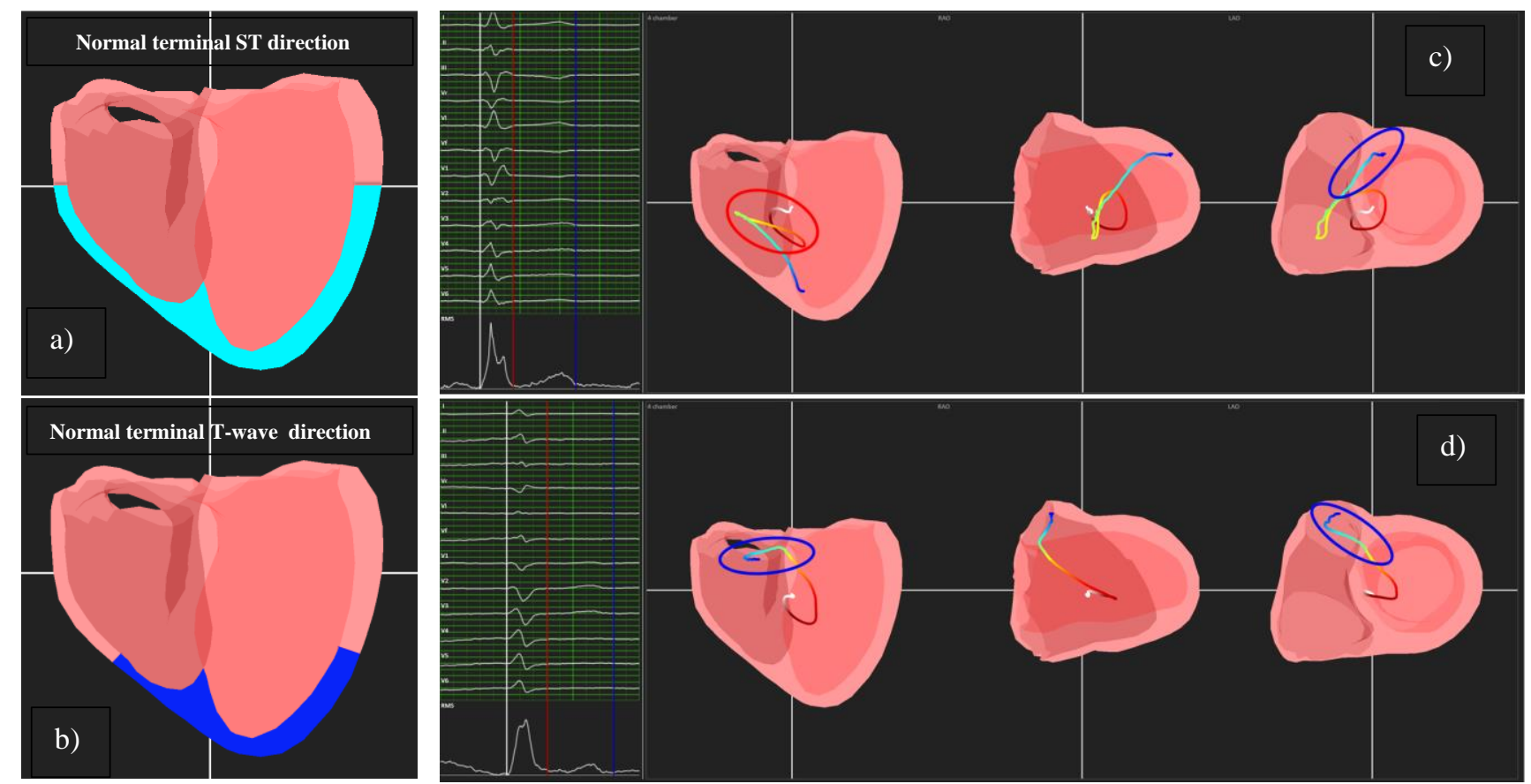

Figure 2: The normal terminal ST (panel a) and terminal T-wave (panel b) directions of the mTSI. When the mTSI terminal directions of a COVID patient was found outside this direction, the ECG beat was classified as abnormal. panel c) visualizes the mTSI pathway from a patient without cardiac history. Delayed QRS activation can be observed towards the right chamber with a large trans-cardiac ratio of more than 37\%, terminal ST and T-wave direction classified as normal. The patient in panel d) shows a more compact mTSI QRS pathway classified as normal, but the terminal ST- and T-wave directions are now far from normal, consequently the ECG beat was classified as abnormal. Deviating part of the

wave $_{\text {peak }}$ ) and the terminal $T$ wave direction ( $\mathrm{T}$-wave peak end) were computed. For every normal control from the PTB-XL database the mTSI and ECG parameters were computed for up to 8 ECG beats with a similar morphology. The range of each of these normal parameter values determined the normal range used in the analysis.

\section{Results}

The ranges of the ECG and mTSI parameters from the normal controls (PTB-XL [3]) were used to classify the QRST wave morphology as normal or abnormal, being:

- $\quad$ QRS duration: $\leq 104 \mathrm{~ms}$

- $\quad$ Trans-cardiac ratio $<37 \%$

- $\quad$ ST ratio: $\leq 0.33$, i.e. peak $T$-wave $>3 \cdot$ ST amplitude

- Terminal ST direction toward the lower apical half of the ventricles, see Figure 2 a for the indicated area in cyan.

- Terminal T-wave direction towards the septal apex, see Figure $2 b$ for the indicated apical area in blue.

When any of the selected parameter values was outside the normal range listed above, the ECG was classified as abnormal. The CineECG classification of either normal or abnormal was compared to the classification from the Capacity-COVID database. The results and the CineECG parameters used to classify the ECG are listed in Table 1. One out of 43 COVID-19 normal ECGs was classified as abnormal, and for 4 ECG of 64 abnormal ECG the classification was normal. Examples of abnormal mTSI paths in Figure 2.

Table 1 CineECG classification of 107 ECG's from COVID-19 patients based on the ventricular waveform of the ECG beats. All parameter values given in \%. Some of the classifications overlap, e.g. the ST direction can be normal and T-wave direction abnormal, or both can be abnormal. Therefor only the total percentage per ECG groups add up to $100 \%$. TCR = trans-cardiac ratio.

\begin{tabular}{|c|l|c|c|c|c|}
\hline \multirow{2}{*}{$\begin{array}{c}\text { ECG } \\
\text { class }\end{array}$} & \multicolumn{1}{|c|}{$\begin{array}{c}\text { Classification } \\
\text { Parameter }\end{array}$} & \multicolumn{2}{c|}{ Normal ECG } & \multicolumn{2}{c|}{ Abnormal ECG } \\
\cline { 3 - 7 } & $\begin{array}{c}\text { No } \\
\text { History }\end{array}$ & $\begin{array}{c}\text { Cardiac } \\
\text { History }\end{array}$ & $\begin{array}{c}\text { No } \\
\text { History }\end{array}$ & $\begin{array}{c}\text { Cardiac } \\
\text { History }\end{array}$ \\
\hline \multirow{5}{*}{ Normal } & QRS $(\leq 104 \mathrm{~ms})$ & 100 & 100 & 41 & 32 \\
\cline { 2 - 7 } & Terminal ST direction & 100 & 100 & 64 & 76 \\
\cline { 2 - 7 } & Terminal T-wave direction & 97 & 100 & 64 & 32 \\
\cline { 2 - 7 } & Total & $\mathbf{9 7}$ & $\mathbf{1 0 0}$ & $\mathbf{5}$ & $\mathbf{8}$ \\
\hline \multirow{5}{*}{$\begin{array}{c}\text { Ab - } \\
\text { normal }\end{array}$} & (I)RBBB & 0 & 0 & 36 & 20 \\
\cline { 2 - 7 } & QRS (>104 ms) & 0 & 0 & 51 & 60 \\
\cline { 2 - 7 } & TCR (>37\%) & 0 & 0 & 21 & 36 \\
\cline { 2 - 7 } & Terminal ST direction & 0 & 0 & 31 & 20 \\
\cline { 2 - 7 } & Terminal T-wave direction & 3 & 0 & 33 & 80 \\
\cline { 2 - 7 } & Total & $\mathbf{3}$ & $\mathbf{0}$ & $\mathbf{9 5}$ & $\mathbf{9 2}$ \\
\hline \multicolumn{2}{|c|}{ Total number ECGs } & 34 & 9 & 39 & 25 \\
\hline
\end{tabular}




\section{Discussion}

This feasibility study shows that the CineECG might be able to classify ECG waveforms to normal or abnormal, a much needed feature required in the critical unit to identify COVID-19 patients at cardiovascular risk. The CineECG classification was derived from 5 parameters, QRS duration, ST ratio, trans-cardiac ratio, and the terminal ST and T-wave direction. The last 3 CineECG parameters are novel as they classify the activation and recovery pathway (mTSI) in relation to the cardiac anatomy, which prove to be able to identify normal from abnormal QRS complex and T-waves (Table 1, Figure 2).

In a previous study we have shown that the mTSI of the QRS and early ST segment can be used to identify Brugada patients due to the terminal QRS direction towards the RVOT. The initial results of study show that this can also be used for the recovery of the heart, the T-wave. The Twave direction of the normals is remarkably consistent in that it follows more or less the septum of the generic heart model, even when most probably the normal subjects must have had a different body build and thus a different heart orientation. A part of the variation of the T-wave direction in the normal population is therefore also attributed to this variation in heart orientation. Further study, using patient specific models of the heart is required to quantify this variation, and to confirm that the normal mTSI direction follows the septum.

The terminal ST direction is less specific than the terminal T-wave direction. As indicated in the Brugada study [5], the ST segment can be used to detect and classify conduction disorders. In this study only the trans-cardiac ratio in combination with the QRS duration (> $104 \mathrm{~ms}$ ) was used to detect evolving conduction disorders in the COVID database. The assumption was that no conduction disorders were present in the normal controls all with a QRS duration of less than $104 \mathrm{~ms}$.

\subsection{Limitations}

The used planes to project the mTSI are dependent on the patient specific orientation and position of the heart in the thorax. For this study, we used a standard heart and thorax model, which influences the results of this study. Moreover, the number of COVID-19 patients included in the study was limited and the background of these COVID19 patients was not always known. Further studies are required therefore.

Also, where CineECG takes patient-specific electrode positioning into account, seriously misplaced 12 -lead ECG electrodes (more than 2-4 cm) might cause misinterpreted mTSI measurements.

\section{Conclusion}

The CineECG is not only able to classify the QRS waveform as normal or abnormal, but also the ST segment and the T-wave. The establishment of the direct anatomical relation in $3 \mathrm{D}$ of the mTSI to the cardiac anatomy might enable the early warning of developing ischemia and myocarditis frequently occurring in COVID-19 patients. The CineECG might thus be a useful tool to screen COVID-19 patients without the immediate need of the expert cardiologist.

\section{Acknowledgements}

This study was funded by Health Holland, project COCVD grant number LSHM 20034 and Dutch Heart Foundation, grants QRS-Vision and eDectect.

Peter van Dam is the owner of ECG Excellence BV and Peacs BV (the Netherlands).

We would like to thank Prof. Dr. FW Asselbergs and MPM Linschoten (UMC Utrecht, the Netherlands) for providing the data from the CAPACITY-COVID collaborative.

\section{References}

[1] Aghagoli G, Gallo Marin B, Soliman LB, et al. Cardiac involvement in COVID-19 patients: Risk factors, predictors, and complications: A review. J Card Surg 2020;35(6):130205. doi: 10.1111/jocs. 14538

[2] Puntmann VO, Carerj ML, Wieters I, et al. Outcomes of cardiovascular magnetic resonance imaging in patients recently recovered from coronavirus disease 2019 (COVID19). JAMA Cardiol 2020 doi: 10.1001/jamacardio.2020.3557

[3] Wagner P, Strodthoff N, Bousseljot RD, Kreiseler D, Lunze FI, Samek W and Schaeffter T. PTB-XL, a large publicly available electrocardiography dataset. Sci Data. 2020;7:154.

[4] van Dam PM. A new anatomical view on the vector cardiogram: The mean temporal-spatial isochrones. J Electrocardiol. 2017;50:732-738.

[5] van Dam PM, Locati ET, Ciconte G, Borrelli V, Heilbron F, Santinelli V, Vicedomini G, Monasky MM, Micaglio E, Giannelli L, Mecarocci V, Ćalović Ž, Anastasia L and Pappone C. Novel CineECG derived from standard 12-Lead ECG enables right ventricle outflow tract localization of electrical substrate in patients with Brugada syndrome. Circulation: Arrhythmia and Electrophysiology. Published online 28 July 2020.

Address for correspondence.

Peter M van Dam

Weiland 38, 2415BC Nieuwerbrug aan den Rijn, the Netherlands

E-mail address: peter.van.dam@ peacs.nl 